\title{
Wie viele Jungs haben Anna geküsst? \\ Zum besonderen Status von V2-Relativsätzen im gesprochenen \\ Deutsch
}

Nicholas Catasso (München)

\begin{abstract}
The aim of the present paper is twofold: first, to introduce the problem represented by socalled "Verb-Second (V2) relative clauses" in German, a phenomenon which occurs exclusively in spoken language, thereby recapitulating the arguments put forth in the literature for their paratactic categorization in light of Den Besten's (1983) assumptions on the structural properties of V2 (e.g. Gärtner 2001, 2002; Den Dikken 2005; Endriss/Gärtner 2005); in the second place, to propose an alternative analysis based on the following postulates: (i) such constructions, which are generally classified as belonging to "noncanonical" phenomena of spoken German, are neither to be ascribed to a deficient syntactic planning nor can they be defined as "false relatives" (cf. Ravetto 2006), but are rather part of a specific syntactic pattern with speaker-oriented implications that is directly related to the syntax-speaker's attitude interface; (ii) these structures display features shared by both asyndetically coordinated clauses (e.g. their word-order and, in principle, the surface form of the $d$-element introducing them) and standard relative constructions, but a paratactic analysis in classical terms (namely one in which it is assumed that clause ${ }_{1}$ and clause 2 are two conjuncts of a coordinative pattern playing the same syntactic role) does not explain most of the distributional properties that "V2 relative clauses" exhibit. The conclusions of the analysis proposed in this paper rest upon evidence of prosodic, syntactic and semantic-pragmatic nature.
\end{abstract}

\section{Standard-Annahmen der syntaktischen Theorie: Das Problem}

Die syntaktische Struktur des Standard-Deutschen weist eine Distributionsasymmetrie des Finitums in Hauptsätzen und Nebensätzen auf, oder, mit Gärtners (2002: 22) Worten ausgedrückt, die Position der Flexion im deutschen Satz "ist sensibel in Bezug auf die Hauptvs. Nebensatz-Differenzierung": Matrixstrukturen zeigen eben eine sogenannte Verb-ZweitWortstellung (V2), wo das flektierte Verb - nämlich das Hilfs- bzw. das lexikalische Verb an zweiter Satzstelle steht, unabhängig von der syntaktischen Natur oder Funktion des Elements in erster Position (Topik/Fokus, Subjekt/Objekt, Komplement/Adjunkt, usw.). Andererseits haben COMP-eingeleitete kanonische Nebensätze, z. B. weil-Sätze und dassSätze, sowie Relativsätze, die aus diachroner Perspektive konservativere VL- bzw. SOV- 
Wortabfolge beibehalten, die sich durch die fixe Stellung des Finitums rechts des Objekts ${ }^{1}$ oder, falls das Tempus analytisch markiert ist, rechts des lexikalischen Verbs, charakterisiert. Ein äußerlich offensichtlicher und umso relevanterer Aspekt unserer Diskussion ist die empirische Tatsache, dass die V2-Wortstellung auch Sätze charakterisiert, die koordinativ miteinander verknüpft sind, wie in Der Hund hat in die Wohnung gemacht und Maria hat ihn ausgeschimpft, d. h. in einer Äußerung, die sich in Hinsicht auf die Sprache der klassischen bzw. symbolischen Logik in Form von $\mathrm{A} \wedge \mathrm{B}$ ausdrücken lässt. Die Asymmetrie zwischen Haupt- und Nebensätzen ist in (1) aufgezeigt:
a. Meine Mutter ruft $[+$ FIN] mich jeden Tag an.
b. Meine Mutter hat ${ }_{[+F I N]}$ mich heute schon dreimal angerufen [-FIN].
c. Ukrainisch ist die Sprache, die mir am besten gefällt $[+F I N]$.
d. Ich habe die Abbiegung genommen, die ich nehmen $[-F I N]$ wollte $[+F I N]$.

Die Beispiele in (1a) und (1b) zeigen, dass in Matrixstrukturen wie unmarkierten Deklarativsätzen das finite Verb unabhängig von der synthetischen (1a) vs. analytischen (1b) Realisierung der Verbalkonstruktion immer an zweiter Stelle vorkommt, während eingebettete Strukturen wie Relativsätze (1c) und (1d) eine Verb-Letzt-Stellung aufweisen.

Den Besten (1983) formalisiert die V2-Wortstellung, die man im Deutschen (sowie im Niederländischen) beobachten kann, indem er das Phänomen mit drei strukturellen Hauptmerkmalen assoziiert: (i) Subjekt-Verb-Inversion; (ii) die zweite Satzposition des Finitums in Matrixsätzen; (iii) die Tatsache, dass diese "linear restriction" ausschließlich in Hauptsätzen vorkommt (was es als "root phenomenon" qualifiziert). Die letztere Eigenschaft, die in normativen Ansätzen noch als begründet gilt, stellt tatsächlich ein Problem für die Einstufung von Nebensatztypen dar, die ursprünglich - wie ihre eingebettete Natur verlangt eine SOV-Wortabfolge haben, in denen aber das Finitum unter bestimmten Bedingungen und nur im gesprochenen Gebrauch an zweiter Satzposition erscheinen kann, wie in folgenden Beispielen:
a. Ich übe deutliche Kritik ganz einfach deshalb, weil wir sind die C-Partei [...].2 (weil-Satz)
b. Ich weiß, dass Herr Laack hat eine Stiftung gegründet. ${ }^{3}$ (dass-Satz)
c. Apfeldorf hat viele Häuser, die stehen leer. ${ }^{4}$ (Relativsatz)

Wie man in (2) sehen kann, ist das nicht-kanonische Phänomen der V2-Einbettung schon in Bezug auf Adjunkt- oder Adverbialsätze (weil-, sowie während-, wobei- und obwohl-Sätze; vgl. z. B. Freywald 2009; Antomo/Steinbach 2010; Gärtner/Michaelis 2010; Antomo 2012), Argumentsätze (vom Komplementierer "dass" eingeleitete Strukturen; vgl. Freywald 2008; 2009) und Relativsätze (vgl. u. a. Curme 1922; Sandig 1973; Gärtner 2001, 2002; Ravetto 2006) beobachtet worden. Solche Strukturen kommen nicht selten, wenn auch mit

\footnotetext{
1 "Objekt" wird natürlich als Sammelbegriff verwendet für alle Elemente, die nicht das Subjekt sind (direkte und indirekte Objekte, Temporal- bzw. Lokalangaben, Subjekts- bzw. Objektsprädikative, usw.).

2 Aus: Norbert Röttgen hatte wirklich eine zweite Chance verdient, 18.05.2012 (Radio-Interview), http://www.dradio.de/dlf/sendungen/interview_dlf/1760319/.

3 Vgl. Freywald (2008: 246).

${ }^{4}$ Vgl. Ebert, Endriss \& Gärtner (2007: 417 ff.).
} 
unterschiedlicher Häufigkeit, in der gesprochenen Sprache vor, und scheinen in Hinsicht auf die grundlegende Differenzierung Haupt- vs. Nebensatz besonders problematisch zu sein. Unter Bewusstwerdung der Standard-Struktur des Deutschen kommt eben die Frage auf, ob sie in Anbetracht von den Bestens (1983) Generalisierung als Matrixsätze oder eher als eine besondere Art von Nebensätzen zu klassifizieren sind. In der vorliegenden Arbeit werde ich mich auf sogenannte V2-Relativsätze konzentrieren und Argumente prosodischer und semantischer Natur für ihren eingebetteten Status vorführen.

\section{Der besondere Status von Relativsätzen}

Die Beispiele in (2a) und (2b) oben betreffen Strukturen, die grundsätzlich zwei syntaktische Eigenschaften besitzen: Sie werden von einem Komplementierer eingeleitet und kommen in der gesprochenen Sprache in zwei Varianten vor (nämlich mit V2- oder VL-Wortstellung). Der letzte Fall, der hier untersucht wird, ist der von Relativsätzen (laut Birkner [2008: 101] dem häufigsten Nebensatztyp), die auch zwei syntaktische Typen erlauben, aber von einem Element eingeleitet werden, das sie von weil- und dass-Sätzen wesentlich differenziert. Die syntaktische Rolle eines Relativsatzes besteht darin, einen nominalen Ausdruck zu modifizieren: Z. B. schränkt der Relativsatz in (3a) die unbestimmte DP "einen Mann" ein, d. h. er liefert zusätzliche Informationen (im weiteren Sinne) zur Beschreibung des Mannes, den Maria kennengelernt hat (und der dem Gesprächspartner offensichtlich unbekannt ist). Wie schon erörtert, unterscheiden sich weil- und dass-Sätze darin, dass es sich dabei jeweils um Adverbial- und Argumentsätze handelt: Erstere sind somit fakultativ und valenzfrei und realisieren keine Theta-Rolle, letztere erfüllen hingegen die Funktion eines Komplements zum Verb (oder zum Adjektiv/Nomen) und sind obligatorisch. Relativsätze spielen in diesem Rahmen eine besondere Rolle, die sie von anderen Nebensatztypen grundsätzlich unterscheiden. Vgl. folgende Beispiele:

(3) a. Die Maria hat einen Mann[AKK] kennengelernt, der[NOM] aus Niederbayern kommt.

b. Prof. Müller hat in Berlin einen Vortrag[AKK] gehört, den[AKK] er nicht vergessen kann.

c. Das ist der Stock[NOM], mit dem[DAT] unser Vater uns immer schlug.

Krifka (2006) identifiziert zwei Perspektiven der syntaktischen Modifizierung einer DP durch einen Relativsatz, nämlich eine Außen- und eine Innenperspektive, die auf die jeweiligen Fragen antworten, wie sich die Relativstruktur auf das Nomen bezieht, das sie modifiziert und wie die modifizierte DP innerhalb des Nebensatzes dargestellt wird. Im Deutschen wird Krifkas "Außenperspektive" durch Phi-Kongruenz und nachgestellte Positionierung realisiert, während die "Innenperspektive" durch die syntaktische Bewegung des Objekts und die entsprechende Leerstelle im Relativsatz ausgedrückt wird - z. B. kann der Relativsatz in (3b) in dieser Hinsicht wie folgt schematisch dargestellt werden: [RS den er _ nicht vergessen kann ]. In den beiden Sätzen in (3a) und (3b) trägt das Relativpronomen, das den Nebensatz einleitet, die Genus- und Numerus-Merkmale der im Hauptsatz vorkommenden DP ("ein Mann" [+MASK; +SING] in (3a) und "ein Vortrag" [+MASK; +SING] in (3b)) und den Kasus, der ihrer Funktion im eingebetteten Satz entspricht (Nominativ in (3a), Akkusativ in (3b)). (3c) zeigt hingegen, dass innerhalb eines komplexeren Relativpronomens wie "mit dem", das eine Präposition enthält, das d-Element in dem von der Präposition verlangten Kasus steht 
(übrigens stellt die auf D-Struktur im Mittelfeld basisgenerierte Konstituente "mit dem Stock" hier ein Adjunkt dar). Relativsätze sind unter den Satztypen, die das V2-Phänomen erlauben, aus vielen Gründen interessant: In erster Linie sind die Relativpronomina "der", "die" und "das" offensichtlich keine Komplementierer: Sie sind immer für das Genus und den Numerus ihres Antezedenten dekliniert und tragen den morphologischen Kasus, der ihre syntaktische Funktion im Nebensatz ausdrückt, sind in den Paradigmen formal identisch mit Demonstrativa - und, als Folge eines Grammatikalisierungsprozesses, der schon im Althochdeutschen begann, mit definiten Artikeln.

\section{Relativsätze mit VL- und mit V2-Wortstellung}

Der Fall der Relativsätze mit oder ohne V2-Wortabfolge ist schon an sich eine Besonderheit, denn solche Konstruktionen werden nicht von einem Komplementierer eingeleitet und weisen trotzdem in kanonischen Kontexten die typische innere Struktur eines Nebensatzes auf. Was hier aber wichtig ist, ist, dass unter bestimmten Bedingungen auch Relativsätze eine V2Wortstellung lizenzieren, wie in (4) gezeigt wird (aus Gärtner [2002: 33] entnommen und angepasst):

(4) a. Das Blatt hat eine Seite, die ganz schwarz ist.

b. Das Blatt hat eine Seite, die ist ganz schwarz.

Beide Sätze in (4) sind grammatische Strukturen des Deutschen, die zusammen mit V2-weilSätzen keinen sonderlich kontroversen Fall darstellen. Natürlich ist eine Äußerung wie (4a) sowohl in der gesprochenen Sprache als auch im Schriftlichen möglich, während die Verwendung einer Struktur wie (4b) auf die mündliche Sprache beschränkt ist. Es bleibt festzustellen, ob und inwiefern sich diese zwei syntaktischen Optionen semantischpragmatisch unterscheiden. Anscheinend betrifft die V2-Variante möglicherweise jede syntaktische Funktion, die von einem Standard-Relativsatz erfüllt werden kann: Das Relativpronomen kann zwar das Subjekt, das direkte bzw. indirekte Objekt, ein lokales bzw. thematisches Argument oder, wie in (3c) gezeigt, ein Adjunkt des Nebensatzes sein. Vgl. z. B. folgende Beispiele:

a. Du weißt Sachen, über die hab ich noch nicht mal nachgedacht. ${ }^{5}$

b. Schau doch mal auf der Homepage, da ist eine Kontaktadresse, über die bin ich gegangen. ${ }^{6}$

Wenn man die semantischen Relationen bzw. die Theta-Rollen der DPs in den zwei Sätzen in (5) in Betracht zieht, sieht man, dass die Konstituenten, die die beiden Relativpronomina auf S-Struktur ersetzen, für Argumente des Verbs stehen, die sich semantisch in unterschiedlicher Weise charakterisieren: in (5a) drückt das Relativpronomen ein thematisches Verhältnis aus (d.h. das von der Präposition "über" eingeleitete Relativpronomen trägt die Theta-Rolle THEMA, die vom Verb "nachdenken" verlangt wird), während im zweiten Fall das Relativpronomen eine lokale Relation zum Ausdruck bringt (was eben durch die Theta-Rolle

5 Aus: http://beautyjunkies.inbeauty.de/forum/archive/index.php/t-58822-p-61.html, Bärbel Drexel, 18.11.2008, Stand: 01.12.2012.

${ }^{6}$ Aus: http://www.wallstreet-online.de/diskussion/798193-1-10/sind-jetzt-endlich-alle-aus-kinowelt-raus, Sind jetzt endlich alle aus Kinowelt raus?, 21.11.2003, Stand: 29.12.2012. 
LOKATIV formalisiert werden kann). Dies deutet darauf hin, dass auch in diesem Fall das Phänomen der V2-Einbettung im Prinzip in allen semantischen Kontexten der Relativsätze erscheinen darf. Wie aber Gärtner (2002: 37) hervorhebt, sind V2-Relativsätze aus syntaktischer Sicht bestimmten Beschränkungen unterworfen, die im Folgenden zusammengefasst und besprochen werden. In erster Linie können sie nur Indefinite modifizieren, d. h. der Kopf des Relativsatzes muss von einem unbestimmten, existentiellen oder von einem ø-Artikel regiert werden. Vgl. (6):

(6) a. Ich habe einen Hund, der hat einen russischen Namen. Die Leute gucken immer komisch, wenn ich ihn rufe: "Bleibda, komm' her!". ${ }^{7}$

b. Apfeldorf hat viele Häuser, die stehen leer. ${ }^{8}$

c. Ich kenne Leute, die haben von diesem Geschäft definitiv mehr Ahnung aufgrund ihrer Berufs- und Lebenserfahrung. ${ }^{9}$

Die Beispiele in (6) sind Relativsätze, die jeweils als Komplement einer unbestimmten DP ("ein Hund"), eines existentiell quantifizierten Nomens ("viele Häuser") und einer DP mit Nullartikel ("Leute") fungieren. Alle DPs stehen hier im Akkusativ und sind ihrerseits Komplemente des Verbs "haben" in den ersten zwei Fällen und des Verbs "kennen" im letzten Beispiel. Diese sind genau genommen typische Verben, die einen V2-Relativsatz einleiten.

An diesem Punkt kommt die Frage auf, ob sich die Sätze in (6) als parataktisch verbunden analysieren lassen. Insbesondere scheint die Hypothese theoretisch plausibel, dass z. B. (6a) und (6b) ungefähr wie folgt umformuliert werden können:

(7) a. Ich habe einen Hund, und der [+DEMONSTRATIV; = der Hund, den ich gerade erwähnt habe] hat einen russischen Namen.

b. Apfeldorf hat viele Häuser, und die [+DEMONSTRATIV; = die Häuser, die ich gerade erwähnt habe] stehen leer.

Strukturen wie (7a) und (7b), die z. B. in Gärtner (2002: 34) und Ebert, Endriss und Gärtner (2007: 416) als "integrierte Verbzweitsätze" definiert werden, tragen diesen Namen im Licht ihrer zweiten fundamentalen Eigenschaft, nämlich die Natur der prosodischen Grenze zwischen dem Hauptsatz und dem Nebensatz. Gärtner nimmt in dieser Hinsicht an, dass es je nach der strukturellen Interpretation des Relativsatzes vier Möglichkeiten gibt: (i) der Relativsatz hat eine Standard-VL-Wortstellung und zwischen dem Haupt- und dem Nebensatz ist eine nicht-finale $[(/)]$ phonologische Pause zu finden; (ii) der Relativsatz hat eine StandardVL-Wortstellung und zwischen dem Haupt- und dem Nebensatz gibt es eine KommaIntonation; (iii) die zwei Sätze werden von einer Komma-Intonation getrennt und die zweite Konstruktion ist nicht ein Relativsatz, sondern er wird als ein normaler Deklarativsatz interpretiert; (iv) die zwei Sätze werden von einer Komma-Intonation getrennt und der von einem Relativpronomen eingeleitete Satz wird als appositiver Satz interpretiert. Solche

\footnotetext{
7 Aus: http://www.kaninchentreff.de/yabbse/index.php?topic=48927.10;wap2, Mein Kamikazehäschen, 30.11.2007, Stand: 21.12.2012.

8 Vgl. Ebert, Endriss \& Gärtner (2007: 417 ff.).

9 Aus: http://www.musiker-board.de/gesangspraxis-voc/488999-mein-traum-ist-musikkarriere-starten-doch-mussich-dafuer-tun-2.html (Musiker-Board, 31.07.2012, Stand: 04.08.2012).
} 
interpretative Unterschiede werden in (8) gezeigt, was tatsächlich zu einem der in der Fachliteratur verbreitetesten Beispiele dieser Erscheinung geworden ist (aus Ebert, Endriss und Gärtner [2007: 416] entnommen und angepasst):
a. Apfeldorf hat viele Häuser, (/) die stehen leer.
b. Apfeldorf hat viele Häuser, (/) die leer stehen.
c. Apfeldorf hat viele Häuser. ( () Die stehen leer.
d. Apfeldorf hat viele Häuser, ( $($ ) die leer stehen.

Die beiden Schreibweisen (/) und $(\backslash)$ deuten jeweils an, ob der Nebensatz eine nicht-finale prosodische Grenzmarkierung (vgl. Gärtner [2002: 104] und Endriss/Gärtner [2005: 196]) oder eine nicht-integrierte Prosodie anzeigt. Obwohl der Status einer Struktur wie (8d) pragmatisch fragwürdig klingt (diese vierte Option wird tatsächlich von denselben Autoren in anderen Kontexten ignoriert und von Gärtner [2002: 35] sogar ausgeschlossen) können Relativsätze mit V2-Wortabfolge daher laut dieser Formalisierung in allen phonologischen Kontexten erscheinen, die Standard-Relativsätze charakterisieren, mit unterschiedlichen Interpretationen. Wie Birkner (2008: 131) bemerkt, gibt es in Standard-Relativstrukturen keine phonologische Pause zwischen dem Haupt- und dem Nebensatz; letzterer ist in die Prosodie des Matrixsatzes obligatorisch (vgl. u. a. Gärtner [2002: 34]) integriert. Insbesondere ist (8a) ein sogenannter "unselbständiger Verbzweitsatz" (in Reis' [1997: 129]) oder - in moderneren Worten - "relativischer Verb-Zweit (RV2) Satz" (vgl. Endriss \& Gärtner [2005: 196]), dessen Modell im Folgenden besprochen wird. Das zweite Beispiel (8b) stellt die kanonische VL-Wortstellung eines nicht pragmatisch markierten Relativsatzes dar, während in (8c) zwei getrennte Sätze ausgesprochen werden $-d$. h. die zwei propositionalen Inhalte sind sowohl syntaktisch, als auch wahrheitswertig voneinander unabhängig, was in der Tat auf der prosodischen Ebene durch eine Komma-Pause widerspiegelt wird (der Sprecher drückt zwei Sachverhalte aus: Apfeldorf hat viele Häuser [und] diese Häuser stehen leer). Der Unterschied zwischen (8b) und (8c) liegt darin, dass im letzteren Satz die Tatsache, dass Apfeldorf viele Häuser hat, als gegeben hingenommen wird und die wichtige semantische Implikation ist, dass sie alle leer stehen, während im Standard-Relativsatz in (8b) gesagt wird, dass die bayrische Stadt Apfeldorf eine unbestimmte Anzahl an Häusern hat und dass von diesen Häusern - die normalerweise wie in jeder Stadt bewohnt werden - viele leer stehen. Die Differenzierung wird durch den Parallelismus mit einem anderen Satz wie dem folgenden deutlicher:

a. Auf der Party waren viele Jungs, die Anna geküsst haben.

b. Auf der Party waren viele Jungs. Die haben Anna geküsst.

Diese zwei Sätze werden aus semantischer Sicht - parallel zum Paar (8a) und (8b) unterschiedlich interpretiert. Die bevorzugte Lesart von (9a) impliziert, dass auf der Party njunge Männer anwesend waren, und dass viele von ihnen Anna geküsst haben. Man kann (zumindest im Normalfall) automatisch ausschließen, dass alle jungen Männer, die auf der Party waren, Anna geküsst haben. Die einzige mögliche Interpretation eines Beispiels wie (9b) wäre, dass von allen Männern, die da waren - nehmen wir einmal: zwanzig -, zwanzig Anna geküsst haben. Im Gegensatz zur Lesart von (9a) steht hier außer Frage, dass nur manche der anwesenden Männer - z. B. zehn von zwanzig - Anna geküsst haben (im Sinne von "Auf der Party waren viele Jungs, und sie alle haben Anna geküsst"). Es bleibt daher 
festzustellen, inwiefern sich der Satz in $(9 b)$ - d.i. die V2-Variante desselben Satzes - z. B. von $(8 b)$ und $(8 c)$ unterscheidet.

Laut Brandt (1990: 40) und Gärtner (2002: 35) sind V2-Relativkonstruktionen distributionell gesehen (d.h. im Vergleich zu Standard-Relativsätzen) ausschließlich restriktiv. Dies nimmt Bezug auf die klassische Differenzierung zwischen sogenannten restriktiven und explikativen oder appositiven oder nicht-restriktiven Relativsätzen (vgl. z. B. Fritsch [1990], Frosch [1996:], Krifka [2006], Birkner [2008]). Ein restriktiver Relativsatz verringert die Menge der möglichen Referenzen, auf die die modifizierte DP im Prinzip zutreffen könnte; andererseits geben nicht-restriktive Sätze weitere beschreibende Informationen über die Bezugsgröße, wie in den folgenden Beispielen:

(10) a. Die Stones sind Leute, die haben ihre Ohren überall hingehalten. ${ }^{10}$ (restriktiv)

b. Mit viel Spaß am Musizieren erarbeiten sich die Kinder, die übrigens auf ihrem Bläserklassenintrument Anfänger/innen sein müssen, im wahrsten Sinne des Wortes "spielerisch" viele Fachkenntnisse und jede Menge spannende Orchesterstücke. ${ }^{11}$ (nicht-restriktiv)

Appositive Relativsätze drücken einen Sachverhalt aus, der die Entscheidung des Sprechers widerspiegelt, eine nähere Beschreibung des Nomens zu liefen, die sich unnötig für die Grammatikalität des Satzes ergibt und informationsstrukturell wie eingeklammert ist: Wie Krifka bemerkt (basiert auf Ballweg et al. [1997: 2007]), sind nur explikative Sätze mit Partikeln wie "übrigens" - die generell Nebeninformationen, d.h. einen textpragmatisch weniger relevanten Inhalt, markieren, vgl. Beispiel (10b) - und mit Eigennamen kompatibel (vgl. "Hans, der 1950 geboren wurde, hat die kulturelle Revolution der 60er Jahre erlebt"); außerdem sind diese Konstruktionen mit einer besonderen inneren Prosodie assoziiert, die signalisiert, dass die im Relativsatz ausgedrückte Information "überflüssig" ist im Hinblick auf den thematischen Fokus der Äußerung, und der Haupt- und der Nebensatz werden von einer Komma-Pause getrennt (s. die Diskussion zur vierten Option in (8d)). In solchen Kontexten zeigt sich die Tatsache, dass das moderne geschriebene Hochdeutsch eine Sprache ist, wo die Zeichensetzung nicht die Prosodie der Äußerungen wiedergibt, sondern eher syntax-orientiert ist: Z. B. dient hier ein graphisches Komma der Trennung von zwei Sätzen, unabhängig davon, ob es um einen restriktiven oder explikativen Relativsatz geht. In anderen Sprachen, wie dem Englischen und dem Italienischen, wird die Differenzierung appositive vs. restriktive Relativstruktur jeweils durch die Verwendung und die Nicht-Verwendung eines Kommas zwischen dem Haupt- und dem Nebensatz markiert, die die Natur der phonologischen Pause signalisiert (und deshalb spricht man von "Komma-Intonation", wenn eine längere Pause nach dem Matrixsatz besteht). Vgl. z. B. das Englische This is the man who I talked to at the party (restriktiv) vs. This man, who I have described in detail in my report, comes from Egypt (nicht-restriktiv). Wie man sieht, kommt ein graphisches Komma ones_1545_audio_128k (Wolfgang Niedecken und die Stones - Interview HR-online.de, 12.07.2012, Stand: 01.08.2012).

11 Entnommen aus: http://www.st-angela-schule.de/index.php?option=com_content\&view=article\&id=65\&Itemid=72, Webseite der St. Angela-Schule [Gymnasium und Realschule] - Bläserklasse, Stand: 01.08.2012. 
nur dann vor, wenn der Relativsatz appositiv ist. Genau genommen ist das Phänomen der V2$\mathrm{d}_{[+\mathrm{REL}]}$-Einbettung in nicht-restriktiven Sätzen nicht erlaubt, wie in (11) gezeigt wird:

(11) a. Dort gibt er seine Frau, die (übrigens) sehr schön ist, als seine Schwester aus.

b. *Dort gibt er seine Frau, die ist (übrigens) sehr schön, als seine Schwester aus.

Die obigen Beispiele illustrieren im Grunde, dass V2-Relativsätze nicht in appositiven Kontexten erscheinen dürfen, und dass Elemente wie "übrigens", die eine Kennzeichnung der Appositiven sind, daher nicht in diesen Konstruktionen vorkommen. In der Tat gelten diese Beobachtungen nur dann, wenn man den Satz "die ist (übrigens) sehr schön" in (11b) als Relativsatz kategorisieren will, denn eine Äußerung wie (11b) wäre sowohl in der gesprochenen als auch (z. B. mit dem interpunktiven Kniff der Bindestriche bzw. der Klammern ausgedrückt) in der schriftlichen Sprache möglich, wenn man den genannten Teilsatz als parenthetischen Ausdruck betrachtet, d. h. als eine Nebenbemerkung, die syntaktisch (sowie prosodisch) getrennt ist von der Proposition, die den eigentlichen Sachverhalt übermittelt (vgl. u. a. Blühdorn [2007: 295-296]). In diesem Fall wäre auch die Anwesenheit von der Partikel "übrigens" natürlich legitim und die prosodische Kontur zwischen den beiden Sätzen wäre nicht die einer Hauptsatz-Relativsatz-Struktur. Dazu kommt auch, dass in dieser Perspektive das einleitende Element des Satzes nicht als Relativpronomen, sondern als echtes Demonstrativ funktioniert. Besonders im mündlichen Gebrauch kommen parenthetische Einschübe sehr häufig vor, und können auch thematisch bzw. diskurspragmatisch vollkommen getrennt sein vom Inhalt des Satzes, wo sie linearisiert werden, wie im folgenden Beispiel, wo man annimmt, dass der Sprecher mit einem Gesprächspartner interagiert - seine kommunikative Intention besteht eben darin, die Information zu übermitteln, dass jemand ("er") seine Frau als seine Schwester ausgibt - und plötzlich (d. h. mitten im Satz) eine andere Person ("Maria") vorbeigehen sieht und mit "Oh, hallo Maria!" begrüßt:

(12) a. ...und dort gibt er seine Frau - oh, hallo Maria! - als seine Schwester aus.

Aber zurück zu den distributionellen Eigenschaften der V2-Relativsätze: Es ist aus der Beobachtung der obigen Beispiele in (8)-(11) ableitbar, dass ein weiteres fundamentales Merkmal dieser Strukturen ist, dass sie ausschließlich extraponiert, d. h. im Nachfeld, vorkommen, was tatsächlich auch über dass-Sätze mit V2-Wortstellung gesagt wurde. Folgende Sätze (aus Endriss/Gärtner [2005: 197] entnommen und leicht modifiziert) illustrieren die Nicht-Positionierbarkeit der V2-Sätze in ihrer Basisposition auf S-Struktur:

(13) a. Die Apfeldorfer haben viele Häuser, (/) die heute leer stehen, gebaut.

b. *Die Apfeldorfer haben viele Häuser, (/) die stehen heute leer, gebaut.

Wieder gilt diese Beschränkung nur dann, wenn - in Anbetracht der Tatsache, dass Relativpronomina und Demonstrativa gleichlautend sind - die vom d-Element eingeleitete Struktur als Relativsatz betrachtet wird, denn mit einer anderen prosodischen Kontur (im vorliegenden Fall: Bei Komma-Intonation) könnten sowohl der Satz in (13a) als auch der in (13b) parenthetische Ausdrücke sein. Hier zieht man aber nur Relativsätze in Betracht, und in dieser Hinsicht soll man zwei wesentliche Aspekte berücksichtigen: Erstens muss man - im Gegensatz zu vielen Arbeiten über nicht-kanonische Konstruktionen der gesprochenen Sprache - eingestehen, dass ein Satz wie (13a), wo der Relativsatz auf S-Struktur in seiner 
Basisposition bleibt, an sich stilistisch und pragmatisch geeignet für den geschriebenen Gebrauch ist, aber in der mündlichen Gegenwartssprache nur eine bloße Möglichkeit des Systems darstellt. Dies hat gut etablierte Gründe psycho-kognitiver Natur: In der gesprochenen Sprache tendiert man nämlich - z. B. aufgrund der physiologischen Grenzen des Gedächtnisses und daher aufgrund des Versuchs einer korrekten und klaren Satzplanung auf eine Planung des auszusprechenden Sachverhalts, die in distinkte Kategorien bzw. Satzteile unterteilt ist. Die Extraposition solcher Konstruktionen spielt also eine erhebliche Rolle im mündlichen Gebrauch und das stellt in der vorliegenden Diskussion ein wichtigeres Argument dar als die Ungrammatikalität der Äußerung an sich. Zweitens drücken V2Relativsätze (wie V2-Nebensätze im Allgemeinen) einen Sachverhalt aus, der neuer und informationsstrukturell relevanter ist als der des Hauptsatzes, dem zufolge der Inhalt des Relativsatzes auf S-Struktur ausschließlich am Satzende, also als extraponierte Struktur, erscheinen darf. Ein Argument, das in der Literatur zur prosodischen Differenzierung von VL- und V2-Sätzen hervorgebracht wurde (vgl. z. B. Birkner [2008: 281] sowie Lee [2012: 86]), ist, dass in VL-Relativsätzen eine Pause nach dem Pronomen des Relativsatzes vorkommt, während $\mathrm{d}_{[+\mathrm{REL}}$-eingeleitete V2-Strukturen keine markierte prosodische Grenze in dieser Position aufweisen. In (14) wird Birkners (2008: 281-282) Schematisierung dieser Regelmäßigkeit wiedergegeben:

(14) a. also ich hab=n freund der: äh (-) fett im geschäft is.

b. ich hab $n=$ freund der hat $=$ n motorboot, $(-)$ und da fahren wir immer auf der mosel. ${ }^{12}$

Es ist aber sehr schwierig, solche Tendenzen der gesprochenen Sprache zuverlässig zu bestimmen. Außerdem scheint diese Generalisierung viel zu ehrgeizig zu sein: Relativsätze mit Verbletzt-Wortabfolge können natürlich eine längere phonologische Pause nach dem Relativpronomen aufweisen, aber das ist nicht im Geringsten eine Regelmäßigkeit der Sprache. Die Anwesenheit der Interjektion "äh" nach dem Relativpronomen deutet übrigens auf die Unsicherheit des Sprechers über den Sachverhalt, die Satzplanung oder die lexikalische Wahl des Satzes an, den das Relativpronomen einleitet. Die Pause vor dem eigentlichen Inhalt des Relativsatzes ist also dadurch motiviert, dass sich der Sprecher die Zeit nimmt, um die Struktur des Satzes zu planen; sie hängt also nicht von einer strukturellen Eigenschaft der Relativsätze ab und keine Pause wird in der Regel vor dem Subjekt einer solchen Konstruktion erwartet. Vgl. z. B. folgende Beispiele aus Video-Interviews, die zufällig auf YouTube ausgewählt wurden und für deren Notation Birkners Symbole verwendet werden:

(15) a. Er sagte mir, er habe noch nie einen Künstler=eine Künstlerin gesehen, die=mit einer solchen Leichtigkeit (-) Geige spielt. ${ }^{13}$

b. Das ist eine Erfahrung, die=auch wichtig ist, um ins Nachdenken zu kommen. ${ }^{14}$

12 In Birkners Darstellung deutet das Symbol (-) auf eine Pause an, während das Symbol = signalisiert, dass die zwei Morpheme, zwischen denen es erscheint, schnell hintereinander ausgesprochen werden.

13 Aus: http://youtu.be/hlpQgL1SyPc (Interview mit Anne-Sophie Mutter, 26.06.2012, Stand: 13.08.2012).

14 Aus: http://youtu.be/OHbtOJOx9zo (Interview mit Rudolf Wötzel - „Über die Berge zu mir selbst“ [Integral], 22.04.2009, Stand: 13.08.2012). 
Wie diese zwei Beispiele zeigen, stellt die Pause nach dem Pronomen kein obligatorisches prosodisches Merkmal dieser Strukturen dar. Ähnlicherweise könnte in einem Relativsatz mit V2-Wortstellung unter bestimmten Bedingungen (d. h. aus den gleichen pragmatischen Gründen, die das Phänomen bei VL-Konstruktionen rechtfertigen) eine Pause nach dem Relativpronomen vorkommen. Phonologische Pausen an dieser Stelle bleiben trotzdem in beiden Fällen unerwartete Realisierungen.

An diesem Punkt wird klar, dass ein Parallelismus mit weil- und dass-Sätzen besteht: Man kann annehmen, dass auch in diesem Fall solche Strukturen, die bei V2-Wortabfolge eben nur extraponiert werden können, eine Art von "Kommentar" zum im Matrixsatz realisierten Kopf des Relativsatzes darstellen und daher unbedingt nach dem Hauptsatz ausgesprochen werden müssen. Dazu kommt, dass auch V2-Relativsätze weder im Skopus der Negation noch im Skopus des interrogativen Satzmodus stehen können, wie in (16a)-(16b) (am Beispiel von (10a)) und in (16c) gezeigt wird:

(16) a. *Sind die Stones Leute, die haben ihre Ohren überall hingehalten?

b. *Die Stones sind nicht Leute, die haben ihre Ohren überall hingehalten.

c. *Ich kenne keine Frau, die hat mehr als fünf Kinder

Außerdem können solche Sätze nicht in syntaktischen bzw. semantischen Kontexten erscheinen, in denen der Inhalt des Nebensatzes nicht assertiert wird, beispielsweise bei Relativsätzen, deren Kopf in einem höheren Nebensatz mit konditionalem Wert realisiert wird, denn die Funktion, die die V2-Wortstellung erfüllt, liegt auch hier darin, dass der eingebettete Sachverhalt an und für sich ausgesagt wird. Vgl. folgendes Beispiel aus Gärtner (2001: 102):

(17) *Wenn du eine Frau kennst, die besitzt ein Pferd, dann [...].

Diese Aspekte deuten alle darauf hin, einerseits dass V2-Relativstrukturen assertive Kraft tragen, und andererseits dass V2-Relativsätze in weniger enger Verbindung mit dem Matrixsatz stehen als ihre Standard-VL-Gegenüber und sich daher auch syntaktisch auf einer anderen Ebene darstellen lassen als Standard-Relativsätze. Im Vergleich zu weil- und dassSätzen besteht hier wie gesagt aufgrund der formalen Übereinstimmung von Relativ- und Demonstrativpronomina noch mehr strukturelle Ambiguität, die auch Autoren wie Gärtner zu einem radikalen Wandel in der Untersuchung der V2-Relativkonstruktionen geführt hat. Was die syntaktische Darstellung dieser Konstruktion betrifft, schlägt Gärtner (2001: 99) eine parataktische Analyse vor. In dieser Hinsicht war dieser Autor der erste, der das Phänomen der V2-Einbettung parataktisch behandelt (alle folgenden Analysen von Adverbialsätzen und dass-Sätzen mit V2-Wortstellung (Steinbach/Antomo [2010]; Antomo [2012]; Freywald [2008; 2009]; usw.) wurden nach diesem theoretischen Modell gebildet. In Abbildung 1 wird die parataktische Annahme illustriert, sowie sie in Gärtner (2001) am Beispiel des Satzes "Das Blatt hat eine Seite, die ist ganz schwarz" erörtert wird: 


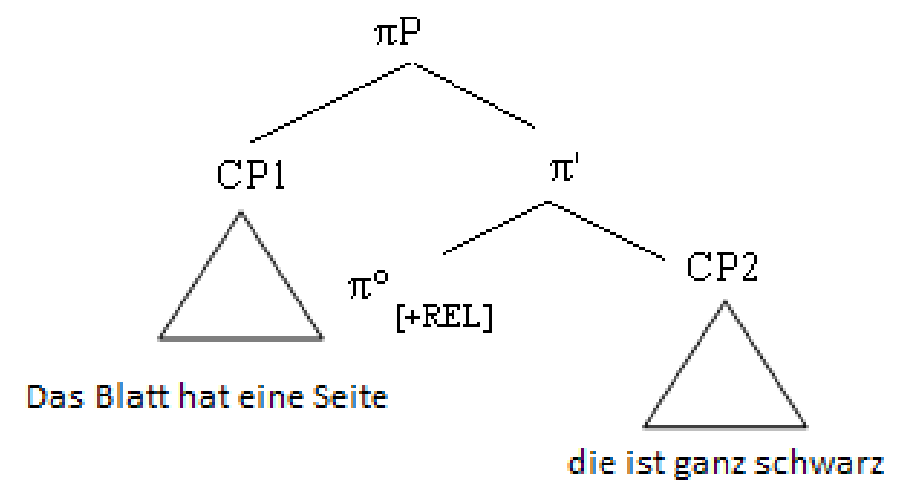

Abbildung 1: Parataktische Darstellung der V2-Relativsätze in Gärtner (2001)

Das in Abbildung 1 dargestellte Modell wird dadurch empirisch begründet, dass V2Relativsätze obligatorisch extraponiert werden und daher immer am rechten Rand des ehemaligen Hauptsatzes vorkommen (vgl. auch Endriss/Gärtner [2005: 197]), was eine parataktische Verbindung ohne Ausnahme garantiert. Außerdem wird das Element, das den Spezifikator von CP2 besetzt, nicht als Relativpronomen, sondern als schwaches Demonstrativ betrachtet. Dieses Argument scheint im Licht der schon erwähnten Übereinstimmung der beiden Kategorien der Demonstrativ- und der Relativpronomina plausibel, aber nicht völlig überzeugend. In Anbetracht der Tatsache, dass parenthetische Ausdrücke im mündlichen Gebrauch sowohl mit Komma-Intonation als auch mit nicht-finaler phonologischer Pause realisiert werden können, argumentieren Gärtner (2001: 99), sowie Endriss und Gärtner (2005: 197f.), dass beide Sätze in (18) möglich sind:

(18) a. *Die Apfeldorfer haben viele Häuser, (/) die stehen heute leer, gebaut.

b. Die Apfeldorfer haben viele Häuser - (/) die stehen heute leer - gebaut.

Diese Hypothese ist zweifellos vernünftig, und tatsächlich wurde etwas Ähnliches in den vorigen Abschnitten gesagt: In der spontanen - d. h. z. B. nicht in einem empirischen Test elizitierten - gesprochenen Sprache, wo viele Aspekte der Prosodie pragmatisch determiniert werden (Sprechtempo, satzinterne Pausen, usw.), sind die exakten phonologischen Grenzen zwischen zwei Sätzen oder Satztypen sehr schwierig zu bestimmen und zu generalisieren. Während (18a) ungrammatisch ist, weil V2-Relativsätze wie gesagt extraponiert werden müssen, zeigt (18b) allerdings, dass V2 in demselben d-eingeleiteten Satz erlaubt ist, falls die Struktur parenthetisch ist (und zwar unabhängig davon, ob die prosodische Pause zwischen dem ersten und dem zweiten Satz in der Linearisierung final oder nicht-final ist). Wenn das einleitende Element eines Satzes wie "das ist ganz schwarz" ein Demonstrativ und nicht ein Relativpronomen wäre - und wenn die Annahme, dass ein Satz wie (18b) möglich ist, de facto stimmt, dann würde unklar bleiben, was im Allgemeinen einen V2-Relativsatz von einer parenthetischen Phrase unterscheidet. Parenthetische Ausdrücke werden selbstverständlich von schwachen Demonstrativpronomina (und nicht von Relativpronomina) eingeleitet, erlauben eine nicht-finale phonologische Grenze nach dem Hauptprädikat, und ihre einleitenden Elemente können jede syntaktische Funktion erfüllen (Subjekt, direktes und indirektes Objekt, usw.). Vgl. dazu (19):

(19) a. Auf der Zeremonie habe ich eine Frau kennengelernt, (/) die ist sehr kultiviert, und ich möchte sie unbedingt wiedersehen. 
b. Auf der Zeremonie habe ich eine Frau kennengelernt - (/) die ist sehr kultiviert - und ich möchte sie unbedingt wiedersehen.

Wenn man annähme, dass in einer Struktur wie (10a) die zwei Teilsätze parataktisch verbunden sind und dass das d-Element ein schwaches Demonstrativ ist (und entscheidenderweise - unter dem Postulat, dass die Prosodie eines parenthetischen Ausdrucks auch nicht-final sein darf), dann müsste man auch daraus schlussfolgern, dass die deingeleitete Konstruktion in (19a) entweder eine parataktische oder eine parenthetische Phrase ist, denn die möglichen Kontexte, wo die beiden Satztypen erscheinen dürfen, kongruieren miteinander. Außerdem sind in dieser Diskussion genuin pragmatische Beobachtungen nötig: Nicht zu vernachlässigen ist z. B., dass bei der Umformulierung solcher Sätze Strukturen entstehen, die sogar in der gesprochenen Sprache wenig plausibel wären, was Gärtner selbst zugibt $^{15}$. Vgl. z. B. folgende Beispiele aus Endriss und Gärtner (2005: 196):

(20) a. Das Blatt hat eine Seite, (/) die ganz schwarz ist.

b. ??Das Blatt hat eine Seite, ( ) die ganz schwarz ist.

c. Das Blatt hat eine Seite, (/) die ist ganz schwarz.

d. ??Das Blatt hat eine Seite. (\) Die ist ganz schwarz.

Um die parataktische Darstellung der V2-Relativsätze zu stützen, wurde das Argument vorgebracht, dass das Element, das den Satz einleitet, nur und ausschließlich ein d-Pronomen sein darf und nicht z. B. eines der Relativpronomina der "welch"-Familie (vgl. Gärtner [2002: $35])^{16}$. Dies ist aber auch bei anderen V2-Satztypen der Fall: Wenn man z. B. weil-Sätze in Betracht zieht, sieht man, dass unter allen möglichen subordinierenden Varianten von "weil", die auch Kausalität ausdrücken ("da", "dieweil"), nur dieser eine Komplementierer V2-Sätze einleiten darf (vgl. *Der Hans kommt nicht, da er ist krank).

Die Sätze in (20) zeigen, dass nicht alle syntaktischen Varianten qualitativ gleichwertig sind. Insbesondere scheinen die Sätze (b) und (d) schräg zu sein, und zwar aus verschiedenen Gründen: Die prosodische Pause in (20b), einem Standard-Relativsatz mit VL-Wortstellung, ist - unter der Annahme, dass der Satz im gesprochenen Gebrauch gesprochen wird - nicht ungrammatisch, sondern unerwartet. Es ist natürlich möglich, dass eine solche Pause zwischen den zwei Sätzen vorkommt, damit der Sprecher den lexikalischen bzw. syntaktischen Inhalt des Nebensatzes planen kann oder sich einfach die Zeit nimmt, darüber nachzudenken, was er genau sagen will. Die Schrägheit von (20b) hängt also davon ab, dass im normalen Fall die intonatorische Kontur der Äußerung sowie in (20a) ist - was nicht im Geringsten ausschließt, dass (20b) auch eine mögliche Konstruktion der gesprochenen Sprache ist. Andererseits sieht (20d) aus pragmatischer Sicht weniger überzeugend aus: Im Prinzip ist eine Struktur, wo ein Demonstrativpronomen eine in einem höheren Satz realisierte DP wiederaufnimmt, möglich, aber in diesem Fall bleibt ein Satz wie (20d) eine bloße Möglichkeit der Sprache, die so unnatürlich klingt, dass sie kaum grammatisch ist (wenn man unter "grammatisch" versteht, dass eine Struktur Teil eines sprachlichen Systems ist, d. h.

15 Gärtner (2002) ändert seine parataktische Auffassung über das Phänomen der V2-Relativsätze - die übrigens die innovativste Idee im Rahmen des generativistischen Ansatzes zur V2-Einbettung dargestellt hatte - und nimmt an, dass V2-Relativsätze existieren.

16 Bezug nehmend auf die Sätze in (21) vgl. z. B. لDas Blatt hat eine Seite, die ist ganz schwarz vs. *Das Blatt hat eine Seite, welche ist ganz schwarz. 
eine Rolle in der Sprache spielt). Syntaktisch gesehen können jeweils (20a) und (20b) einerseits und (20c) und (20d) andererseits zusammen eingestuft werden: Im ersteren Fall haben die zwei Sätze eine VL-Wortstellung (d.i. die, die der deutschen Sprachnorm entspricht), während bei (20c) und (20d) das Finitum an zweiter Stelle steht. Den Unterschied macht offensichtlich die Prosodie aus, insbesondere die Pause zwischen den zwei Sätzen (und daher die Kategorisierung des Elements, das den zweiten Satz einleitet). An diesem Punkt kommen die Fragen auf, warum ein Satz wie (20d) schräg klingt, während sein Gegenüber (8c) ("Apfeldorf hat viele Häuser. (\) Die stehen leer"), das strukturell identisch ist, als völlig grammatisch anerkannt wird, und warum (20d) pragmatisch weniger plausibel als (20c) klingt. In erster Linie soll man berücksichtigen, dass die beiden Varianten (8c) und (20d), die aus zwei Sätzen bestehen und wo das einleitende Element des d-Satzes ein schwaches Demonstrativ ist, genau dem entsprechen, was man unter "parataktischer Verbindung" versteht (d. h. zwei CPs, die ohne overt realisierte Koordination nebeneinander in die Struktur gestellt werden und strukturell gleichwertig sind, d. h. zwei CPs, zwischen denen keine subordinative Verbindung besteht). Nichtsdestoweniger - wenn man z. B. (20c) und (20d) in Betracht zieht - muss man feststellen, dass die beiden phonologischen Optionen nicht grammatisch gleichwertig sind, weil sie nicht das Gleiche bedeuten. Unbeschadet der pragmatischen Schrägheit von (20d) will man mit diesem Satz nämlich sagen: "Dieses Blatt hat (anders als jedes andere Blatt?) nur eine Seite und diese Seite ist schwarz". Die Äußerung enthält zwei Inhalte, die informationsstrukturell der Art TOPIC-COMMENT + TOPIC-COMMENT gebildet sind, wie folgt:

(21) $[[$ Das Blatt topic] [hat eine Seite comment $] \operatorname{CP(1)}]$. [[Die topic] [ist ganz schwarz COMMENT]CP(2)]

Einer der Gründe, warum (21) pragmatisch fragwürdig klingt, ist, dass im Licht der Darstellung in (22) der erste der zwei Sachverhalte, d. h. dass das Blatt (nur) eine Seite hat, gegen die Gesetze der Logik - und der Physik - verstoßt, denn ein Blatt muss mindestens und maximal zwei Seiten haben, während man in (20d) die Information übermittelt, dass die einzige Seite, die das (schon im Text erwähnte) Blatt hat, ganz schwarz ist. Damit wird klar, dass zwei Konstruktionen wie (20c) und (20d) nicht dieselbe Informationen enthalten und daher nicht auf der gleichen semantisch-pragmatischen Ebene behandelt werden sollen, was auch die unbestrittene Grammatikalität und Plausibilität von (20c) rechtfertigt: Die (/)Variante wird an und für sich als ein Relativsatz interpretiert, wo man den Inhalt ausdrückt, dass von den zwei Seiten des Blatts eine ganz schwarz ist. Wenn die Lesart von (20c) identisch mit der von (20d) wäre, würde auch dieser Satz unlogisch klingen, was aber nicht der Fall ist. Es bleibt trotzdem zu bestimmen, warum (8a) und (8c) beide als völlig grammatisch empfunden werden und daher warum (8c) und (20d) verschiedene Akzeptanzgrade aufweisen. In (22) werden (8a) und (8c) zur Bequemlichkeit des Lesers wiederholt:

(22) a. Apfeldorf hat viele Häuser, (/) die stehen leer.

b. Apfeldorf hat viele Häuser. ( $($ ) Die stehen leer.

Die Tatsache, dass in dieser Perspektive beide Varianten - im Gegensatz zu (20c) und (20d) Konstruktionen des Deutschen sind, hängt vom logischen Status dieser Äußerung ab. Wie oben gezeigt, enthält auch (22b) zwei Aussagen: Man assertiert einerseits, dass Apfeldorf 
viele Häuser hat, und andererseits, dass diese Häuser (d. h. alle Häuser, die im vorigen Satz erwähnt werden) leer stehen. Mit anderen Worten entspricht (22b) der Umformulierung "Alle Häuser in Apfeldorf stehen leer, es ist ein Phantom-Dorf". Die Semantik von (22a) impliziert hingegen, dass von allen Häusern, die sich in Apfeldorf befinden, viele leer stehen. In (22b) liegt also im Gegensatz zu (20d) keine Verletzung der Logik vor, d. h. die zwei Inhalte machen beide Sinn und können vernünftig parataktisch verbunden werden. Was aber hier relevant ist, ist, dass die zwei Sätze in (22) nicht das Gleiche bedeuten, da die d-Struktur im ersten Satz im Allgemeinen nur als Relativsatz interpretiert werden kann. Die zwei in diesem Abschnitt diskutierten Beispiele deuten klar darauf hin, dass die semantisch-pragmatische Intention des Sprechers bei der Produktion eines V2-Relativsatzes nicht parataktisch sein kann. Dasselbe kann daher über das Niederländische gesagt werden, wo das Phänomen der V2-Relativsätze mit ähnlicher Häufigkeit und unter gleichen Bedingungen vorkommt. Vgl. z. B. die Analyse von Den Dikken (2005: 700f.), der das Auftreten von V2 in Relativsätzen durch folgende Beispiele veranschaulicht (der Satz in (23b) ist übrigens parallel zu (22)):

(23) (23) a. Er waren twee jongens op het strand die hadden geen zwembroek aan.

Es waren zwei junge Männer am Strand, die hatten keine Badehose an.

b. Apeldoorn heeft veel huizen die staan leeg.

Apfeldorf hat viele Häuser, die stehen leer.

Die in (23) aufgeführten Beispiele zeigen tatsächlich zwei typische Kontexte, die V2Relativsätze erlauben, nämlich einleitende Prädikate wie "Es gibt + NOMEN $_{\text {[PLURAL] }}+$ Relativpronomen" und "Subjekt + haben + direktes Objekt + Relativpronomen". Den Dikkens Analyse, die die V2-Einbettung in den germanischen Sprachen nur kurz erwähnt und ausschließlich am Beispiel der V2-Relativsätze im Niederländischen untersucht, geht interessanterweise in dieselbe Richtung wie unser Vorschlag für alle ursprünglichen VLSatztypen (Adverbial-, Argument- und Relativsätze), die unter besonderen Bedingungen eine V2-Wortstellung erlauben. Der Autor sieht diese Sätze als die pragmatische Realisierung eines Topik-Kommentar-Modells, wo der Hauptsatz das Bekannte (d. h. den Hintergrund) ausdrückt, während der informationell wesentliche Teil der Äußerung der Relativsatz ist: Wie Den Dikken bemerkt, bringt der Satz in (24a) keine Assertion zum Ausdruck über die Tatsache, dass die gesamte Anzahl der jungen Männer am Strand zwei ist. Man assertiert eher, dass von allen Leuten männlichen Geschlechts, die sich in dem Moment am Strand befanden, zwei keine Badehose anhatten. Der Matrixsatz stellt die Hintergrund-Information dar, die auf jeden Fall nötig für die Wohlgeformtheit der Äußerung ist, aber nicht der relevanteste bzw. informativste Teil des Satzes: In (23a) assertiert der Sprecher durch den vom d-Element eingeleiteten Satz etwas hoch Informatives über das Nomen, das im Hauptsatz realisiert wird und als Kopf des Relativsatzes fungiert. Auch im Niederländischen in Bezug auf (23b) - gelten dieselben Beobachtungen, die in der Diskussion zu (22) angeführt wurden: In (23b) wird keine Aussage gemacht, dass Apeldoorn viele Häuser hat, sondern der Sprecher assertiert etwas über viele - aber nicht alle, wie im Hauptsatz ausgedrückt - der Häuser, die sich da befinden. Solche semantischen Implikationen schließen automatisch aus, dass es sich dabei um eine Parataxe (zumindest um eine Parataxe im klassischen Sinne) handeln kann. Den Dikken ist trotzdem der Ansicht, dass sich diese Konstruktionen nicht als "echte" Relativsätze analysieren lassen, weil - wie er auf Empfehlung von Anikó Lipták (vgl. 
Den Dikken 2005: 700f.) bemerkt - es nicht mehr als eine V2-Relativstruktur in einem Satz geben darf, wie (24) illustriert:

(24) a. Apeldoorn heeft veel huizen die leeg staan die geen hond wil kopen. Apfeldorf hat viele Häuser, die leer stehen, die kein Schwein (= Hund) kaufen will

b. *Apeldoorn heeft veel huizen die staan leeg die wil geen hond kopen. Apfeldorf hat viele Häuser, die stehen leer, die will kein Schwein (= Hund) kaufen

Dies kann auf die Tatsache zurückgeführt werden, dass V2-Nebensätze innerhalb des TopikKommentar-Schemas die neuere, relevantere Information realisieren. In einer einzelnen Äußerung kann es tatsächlich nur eine neue Information geben, die im Übrigen nach dem Topik erscheint. In Beispiel (24a), das eine kanonische Standard-Wortstellung aufweist, ist die informationell wichtige Information, dass niemand die genannten Häuser in Apeldoorn kaufen würde, nicht, dass viele Häuser in Apeldoorn leer stehen. Der Relativsatz "die geen hond wil kopen" bezieht sich auf die ganze Struktur "Apeldoorn heeft veel huizen die leeg staan" (die eben auch den Relativsatz "die leeg staan" enthält) und stellt das Neue der Äußerung dar. Es ist also überhaupt nicht überraschend, dass zwei nicht koordinierte V2Relativsätze innerhalb derselben Konstruktion zu Ungrammatikalität führen. Außerdem realisieren das Neue und das Bekannte in jeder sprachlichen Konstruktion eine verschiedene prosodische Kontur. Insbesondere wird die neue Information mehr betont als die schon bekannte, und wenn in einer Äußerung zwei (benachbarte) Sätze "das Neue" darstellen würden, dann würde die prosodische Kontur der gesamten Struktur abweichend werden. Ein fundamentaler Aspekt der Frage, den Den Dikken nicht erwähnt und der das Argument der "Nicht-Zusammenstellbarkeit" der V2-Relativsätze widerspricht, ist, dass es möglich ist, Strukturen zu haben, wo der V2-Relativsatz wie erwartet am Satzende erscheint und der nominale Kopf schon von einem VL-Relativsatz modifiziert wird, was in (25a) und (25b) gezeigt wird:

(25) a. Es gibt ein paar Leute, die ich kenne, die haben sich hochgearbeitet. ${ }^{17}$

b. Naja, irgendwann habe ich dann jemanden getroffen, der hier arbeitet, der hat mir dann gezeigt, wo die richtige Richtung ist. ${ }^{18}$

c. *Es gibt Jugendliche, die lieben Rock-Musik und Pop-Musik hassen.

d. ??Es gibt Jugendliche, die Rock-Musik lieben und hassen Pop-Musik.

Über die obigen Beispiele kann man viele Beobachtungen anstellen. Erstens zeigen (25a) und (25b), dass der Sprecher in beiden Fällen nicht zwei Inhalte ausdrücken will, sondern eine einzige Assertion macht, die sich in zwei Teile unterteilt: ein Topik und ein Kommentar (insbesondere, eine Hintergrund-Information und eine informationell wichtigere Information). In (25a) und in (25b) will der Sprecher den versprachlichten Sachverhalt nicht wie folgt organisieren:

a. \#Es gibt ein paar Leute, die ich kenne. Die haben sich hochgearbeitet.

17 Aus: http://www.gutefrage.net/frage/1500-netto-ohne-schulabschluss (1500€ netto ohne Schulabschluss? Forum, 25.05.2012, Stand: 12.06.2012).

18 Aus: http://www.myfanfiction.de/texte/taylor-lautner/a-different-way-to-get-big-problems.149201.465441.html (Unglück im Glück? - Fanfiction.de, 2012, Stand: 16.02.2013). 
b. \#Naja, irgendwann habe ich dann jemanden getroffen, der hier arbeitet. Der hat mir dann gezeigt, wo die richtige Richtung ist.

Wenn es so wäre, würde der Sprecher in (25a) zwei Inhalte assertieren: Dass es ein paar Leute gibt, die er kennt, und dass sich diese Leute hochgearbeitet haben. Hier wird aber nicht gesagt, dass von den vielen Bekannten, die der Sprecher in seinem Leben hat, ein paar sich hochgearbeitet haben. Die ganze Struktur klingt in der Tat eher schräg, es sei denn, dass sich der Sprecher und sein Gesprächspartner z. B. auf einer Party befinden und der Sprecher bemerkt, dass da ein paar Leute sind, die er kennt, und den Kommentar hinzufügt, dass sie sich hochgearbeitet haben. Auch unter solchen kontextuellen Bedingungen wäre die Konstruktion aber fragwürdig. Außerdem wäre die Koindizierung des Indefinitpronomens "jemand" und des Demonstrativs "der" in (26b) ziemlich merkwürdig, woraus sich leicht schlussfolgern lässt, dass der vom d-Element eingeleitete Teilsatz nicht ein StandardDeklarativsatz (d. h. ein Satz, der parataktisch verbunden mit dem Rest der Struktur) ist, sondern obligatorisch als Relativsatz interpretiert werden muss (ein Relativsatz, der, wie wir gesehen haben, natürlich einen assertiven Status hat, der durch die V2-Wortabfolge des Nebensatzes kodiert wird). Ohnedies wäre es aus pragmatischer Sicht sonderbar (allerdings unerwartet), dass der Gesprächspartner dem Inhalt des ersten Satzes widersprechen oder ihn hinterfragen würde (Gleiches gilt eigentlich - wie wir im Folgenden sehen werden - für den Sachverhalt des zweiten Satzes). Vgl. die Beispiele in (27):

(27) a. SPRECHER A: Es gibt ein paar Leute, die ich kenne, die haben sich hochgearbeitet. SPRECHER B: ??Nein, du kennst keine Leute, (oder)

SPRECHER B: ?Nein, die haben sich nicht hochgearbeitet.

b. SPRECHER A: Maria ist nicht daheim. Sie muss auf dem Fischmarkt sein. SPRECHER B: Doch, doch! Sie ist zu Hause, ich habe sie gesehen!

Das Beispiel in (27a) soll zeigen, dass der erste Satz "Es gibt ein paar Leute, die ich kenne", der als einleitendes Prädikat des Relativsatzes fungiert, nicht als unabhängige Struktur funktioniert und daher nicht eigenständig bestritten werden kann. Dies gibt eindeutig an, dass das Prädikat nicht parataktisch verbunden mit der folgenden Struktur ist. Wenn es sich dabei um eine echte Parataxe handeln würde, wie bei (27b), wo "Maria ist nicht daheim" und "Sie muss auf dem Fischmarkt sein" zwei Hauptsätze sind, könnte man in Prinzip den ersten Satz widersprechen (s. "SPRECHER B"), denn er wäre syntaktisch getrennt vom folgenden Hauptsatz. Dass die Wahrheit des Nebensatzes in (27a) im Grunde genommen nicht in Frage gestellt werden kann (s. den zweiten "SPRECHER B" in (27a)), deutet darauf hin, dass der Inhalt des Relativsatzes stark sprecher-orientiert ist und nicht eine Aussage darstellt, die sich widersprechen lässt. Die Tatsache, dass die Beispiele in $(25 a) /(25 b)$ im Gegensatz z. B. zu (24b) grammatisch sind, deutet übrigens darauf hin, dass Relativsätze mit V2-Wortstellung nicht die gleiche syntaktische Stelle einnehmen wie VL-Relativsätze, sondern höher in der Struktur positioniert sind. Diese Idee wird in (25c) und (25d) bestätigt: da zwei Sätze mit unterschiedlicher Stellung des Verbs nicht koordiniert werden dürfen, muss man schlussfolgern, dass V2- und VL-Nebensätze miteinander inkompatibel sind, d.h. dass sie in unterschiedlichen Stellen adjungiert werden und daher an der Schnittstelle Syntax-SemantikPragmatik verschiedene Funktionen erfüllen müssen. Traditionell wird im generativen theoretischen Ansatz angenommen, dass restriktive Relativsätze an die NP adjungiert werden, 
worauf sie sich beziehen (d. h. die als ihr struktureller Kopf fungiert), während appositive oder nicht-restriktive Relativsätze an die DP adjungiert werden (vgl. z. B. Sturm [2005: 109f.] und Von Stechov [2007]). Sternefeld [2004: 296] problematisiert jedoch diesen Rahmen, indem er die Hypothese aufstellt, dass "die Verwendungsweise eines Relativsatzes nicht direkt mit einer bestimmten Position korreliert", denn die Relativkonstruktion wird da adjungiert, wo sie "im intendierten Sinne semantisch interpretierbar ist". Der Autor veranschaulicht seine Ansicht mit folgenden Beispielen:

a. ?Der Mann und die Frau, die einander lieben.

b. ?Jeder Kandidat, der ja in der Regel gleichzeitig Mitglied des Gemeinderates ist, hat die Möglichkeit, sämtliche Mitgliedschaften ruhen zu lassen.

Wie Sternefeld bemerkt (vgl. auch die Diskussion in Sturm [2005: 110]), sind die Beispiele in (28) aus theoretischer Sicht problematisch, wenn man die genannte Adjunktionsregel annimmt: Der Satz in (28a), der hier restriktiv interpretiert wird, darf an und für sich nicht an NP adjungiert werden in Anbetracht der Tatsache, dass der Plural des Verbs ("lieben") die Koordination von zwei DPs verlangt. Außerdem käme unter solchen Bedingungen die Frage auf, an welche der zwei NPs "Mann" und "Frau" der Satz adjungiert werden soll - aus Linearisierungsgründen wäre die Antwort "Frau", aber wie gesagt muss der Plural "lieben" auf jeden Fall durch eine DP-Koordination gerechtfertigt werden. Dazu kommt auch, dass in einem Satz wie (28b) das Gegenteil vorkommt: Wenn der Relativsatz an die ganze DP adjungiert wird, dann entstehen semantische Probleme, die damit zu tun haben, dass sich der Relativsatz auf die Eigenschaft [+Kandidat sein] beziehen muss, was tatsächlich nur dann erlaubt ist, wenn der Nebensatz an die NP "Kandidat" adjungiert wird. Sternefelds Analyse setzt also voraus, dass die syntaktische Stelle der Relativsätze - unabhängig davon, ob sie restriktiv oder nicht-restriktiv sind - variabel ist, denn eine feste Kategorisierung wie die, die traditionell angenommen wird, bringt wesentliche semantische Probleme mit sich.

Wenn man eine Struktur wie die in (10a) in ihrer kanonischen VL-Form in Betracht zieht, nimmt man einfach an, dass der Relativsatz als Komplement des Nomens erzeugt und auf SStruktur extraponiert wird. So eine syntaktische Derivation ist unter dem Gesichtspunkt des Zusammenhangs zwischen Haupt- und Nebensatz absolut unproblematisch. "Die Stones sind Leute" ist der Matrixsatz und "die ihre Ohren überall hingehalten haben" ist der restriktive Relativsatz, der das Nomen "Leute" modifiziert und sowohl syntaktisch als auch prosodisch völlig integriert im Hauptsatz ist: Er kann im negativen und im interrogativen Skopus stehen (vgl. $\sqrt{ }$ Die Stones sind nicht Leute, die ihre Ohren überall hingehalten haben, sondern [...];

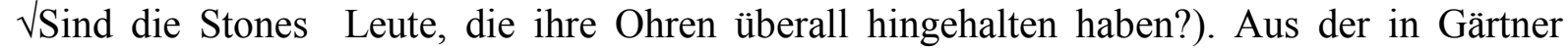
(2001) und Steinbach und Antomo (2010) angenommenen Darstellung desselben Satzes in seiner V2-Variante ersieht man, dass strukturelle und semantische Probleme entstehen. Der dSatz wird laut diesem Modell von einem Demonstrativpronomen eingeleitet und die zwei Sätze sind parataktisch verbunden. Dies würde implizieren, dass der Satz auch formulierbar wäre als "Die Stones sind Leute. Die haben ihre Ohren überall hingehalten", d. h. als ein Satz, der sowohl inhaltlich als auch pragmatisch nicht überzeugend ist. In erster Linie - wie bei den anderen Beispielen, die oben analysiert wurden - assertiert man nicht zwei Inhalte: Man sagt nicht, dass die Rolling Stones Leute (d.h. nicht etwas Anderes) sind und dass die eine 
bestimmte Aktion ausgeführt haben. Übrigens wäre die Verwendung eines Demonstrativs wie "die" in diesem Kontext sehr fragwürdig und die Äußerung würde kaum einen Sinn ergeben, denn es steht außer Zweifel, dass die Stones menschliche Wesen - und nicht z. B. Tiere oder Roboter - sind, also kann der Satz "Die Stones sind Leute" (zumindest unter normalen Bedingungen; allerdings ist die Konstruktion in dem Kontext, aus dem das Beispiel entnommen wurde, ein Relativsatz) keinen möglichen Beitrag zur Konversation leisten. Ein weiterer Aspekt, dessen Wichtigkeit in dieser Diskussion betont werden soll, ist die SemantikPragmatik dieser Strukturen: Wie auch in Hinsicht auf weil- und dass-Sätze mit nichtkanonischer Verbstellung vorgeschlagen wurde, ist es plausibel, dass Strukturen, die in ihren Norm-orientierten, nicht-markierten Instanzen eine VL-Wortabfolge haben und in manchen Fällen auch V2 erlauben, bestimmte semantisch-pragmatisch Werte ausdrücken, die je nach der spezifischen Wortstellung (und - wie wir gesehen haben - auch der satzinternen Prosodie) variieren.

Wenn man davon ausgeht, V2-Relativsätze seien eigentlich Nebensätze und nicht Hauptsätze, kann angenommen werden, dass die Intention des Sprechers in der Verwendung solcher Konstruktionen darin liegt, einen Sachverhalt auszudrücken, der: (i) "neuer" bzw. diskursinformationell relevanter ist als der Inhalt des Matrixsatzes; (ii) assertiert wird (was faktisch die Tatsache rechtfertigt, dass der Matrixsatz kein interrogatives bzw. negatives Prädikat enthalten darf); (iii) sich als das Resultat der emotionalen Verwicklung des Sprechers ergibt: V2-Relativsätze kommen nie in neutralen Kontexten vor - der Inhalt des Nebensatzes ist immer gewissermaßen dem Sprecher "nah" und deshalb sind solche Sätze ausschließlich ein Phänomen der gesprochenen Sprache. Punkt (i) wurde mehrmals in der Erörterung des nicht-parataktischen Status dieser Strukturen demonstriert: In einem Satz wie (25a) ("Es gibt ein paar Leute, die ich kenne, die haben sich hochgearbeitet") ist die im V2-Relativsatz ausgedrückte Information viel wesentlicher als die des Matrixsatzes, was in der Tat einem allgemeinen Satzmodell entspricht, wo das informationell Relevante nach dem ausgesprochen wird, was der Sprecher als Hintergrund-Information konzeptualisiert. Dass sich die Leute, die der Sprecher kennt, hochgearbeitet haben, stellt insofern den Dreh- und Angelpunkt der Äußerung dar und wird deshalb am Satzende gesagt. Was Punkt (ii) betrifft, ist die Ungrammatikalität von V2-Sätzen nach negierten oder interrogativen Prädikaten ein zuverlässiges Indiz dafür, dass der Sachverhalt des Nebensatzes assertiert wird. V2Relativsätze können auch von einem Prädikat wie "suchen" eingeleitet werden und eine Eigenschaft bestimmen, die kontextuell mit der Semantik des Verbs "suchen" verlangt wird (also eine Eigenschaft, die das Kopf-Nomen in der sprachlichen Intention des Sprechers idealerweise haben sollte und noch nicht vorhanden ist). In solchen Fällen ist das Vorkommen von Modaladverbien wie "vielleicht" im Nebensatz ausgeschlossen. Vgl. folgende Beispiele:

(29) a. Ich suche eine Frau, die aus meiner Nähe kommt.

b. Ich suche eine Frau, die kommt aus meiner Nähe.

c. Ich suche eine Frau, die vielleicht aus meiner Nähe kommt.

d. *Ich suche eine Frau, die kommt vielleicht aus meiner Nähe.

Die semantische Nuance, die die Beispiele in (29) involvieren (d. h. dass man von einer "idealen", noch unbekannten Frau redet, die eine bestimmte Eigenschaft haben muss, um die 
Suche des Sprechers zu befriedigen), wird generell in den romanischen Sprachen durch den Konjunktiv Präsens ausgedrückt ${ }^{19}$. Das Paar (29c)-(29d), wo das Adverb "vielleicht" im nicht kategorisch zweifelnden Sinne von "eventuell", "möglicherweise" intendiert wird, zeigt, dass in Anbetracht des [+ASS]-Status des V2-Nebensatzes jedes nicht-assertive Element die Äußerung ungrammatisch macht. Dass der Inhalt des Nebensatzes assertiert wird, sieht man nicht nur an der Wortstellung, sondern auch an der satzinternen Prosodie des Relativsatzes (während die phonologische Grenze zwischen Haupt- und Nebensatz wie gesagt identisch mit der eines Standard-Relativsatzes ist). In dieser Hinsicht kann man im Allgemeinen annehmen (wobei - wie schon in den vorigen Abschnitten erwähnt wurde - keine spezifischen Studien vorliegen, die sich auf die segmentale und suprasegmentale Merkmale der V2-Relativsätze konzentrieren und auf durch spektrometrische Tests erhobene Daten basieren), dass sich die Intonation eines V2-Relativsatzes genauso wie Äußerungen mit Standard-VL-Sätzen als progredient charakterisiert, aber da in solchen Konstruktionen die V2-Wortstellung auch die Einstellung des Sprechers ausdrückt, kann die intonatorische Kontur des Nebensatzes ausdrücklich betont werden (besonders im Vergleich zur Intonation des Hauptsatzes). Dies ist dadurch motiviert, dass der Sachverhalt, der im V2-Relativsatz versprachlicht wird, ausweislich der Intention des Sprechers das Wesentliche seines verbalen Beitrags zum Sprechakt darstellt. In diesem Sinne drückt die syntaktische Auswahl zwischen V2 und VL die Einstellung des Sprechers dem gegenüber, was er sagt, aus: Es ist der Sprecher, der die zu übermittelnden Informationen selegiert, als mehr oder weniger relevant bewertet und eventuell durch bestimmte Mittel des sprachlichen Systems (im vorliegenden Fall: Die Syntax und die Prosodie) betont. Vgl. folgende Beispiele in (30):

(30) a. Bei uns ist ein Mann, der redet einfach drauflos, ob er allein ist oder nicht. ${ }^{20}$

b. Das ist Musik, die wird nicht sterben. ${ }^{21}$

(30a) und (30b) stellen zwei interessante Fälle dar. Abgesehen vom syntaktischen Faktum, dass man im ersteren Satz nicht zwei separate Aussagen (die häufige Anwesenheit eines bestimmten Mannes an einem bestimmten Ort und - als getrennte Information - sein Soliloquium) verbindet, denn der Nebensatz ist syntaktisch in den Matrixsatz integriert, deutet die Tatsache, dass die V2- und nicht die VL-Wortstellung verwendet wird, darauf hin, dass der Sprecher den im Nebensatz ausgedrückten Teil des Satzes als den relevantesten betrachtet, während der Rest nur dazu dient, den Background zur neuen Information zu bilden. Die Einstellung des Sprechers der Tatsache gegenüber, dass der Mann allein spricht, ist nicht neutral: Er findet die durch V2 geäußerte Information entweder hochinteressant oder sehr merkwürdig (in diesem Fall geht es um eine Forum-Diskussion über Schizophrenie), auf jeden Fall muss der Inhalt, der im Relativsatz versprachlicht wird, dem Sprecher nah sein. Gleiches gilt für (30b): der Satz wurde aus einem Interview entnommen, wo ein enthusiastischer Zuschauer eines Abba-Konzerts in Trier seine Eindrücke über die Show und

\footnotetext{
19 Vgl. z. B. Italienisch „Cerco una donna che abiti dalle mie parti“, Französisch „Je cherche une femme qui soit franche et fidèle“, Spanisch „Busco una mujer que le guste bailar“.

20 Aus: http://www.gutefrage.net/frage/leute-die-mit-sich-selber-reden, gutefrage.de - „Leute, die mit sich selber reden “, 15.09.2010, Stand: 10.12.2012.

21 Aus: http://www.volksfreund.de/nachrichten/region/kultur/Kultur-in-der-Region-Das-ist-Musik-die-wird-nichtsterben;art764,3023998, volksfreund.de - „Das ist Musik, die wird nicht sterben“, 09.01.2012, Stand: 12.02.2012.
} 
über die Musik der 70iger Jahre äußert: Es ist klar, dass der Grund, weshalb der Nebensatz eine V2-Wortabfolge aufweist, der ist, dass der Sprecher besonderen Wert auf die Aussage legt, die der Satz übermittelt. Übrigens wäre der Satz - und im Besonderen die Verwendung eines Demonstrativpronomens als einleitendes Element des zweiten Satzes - sehr fragwürdig, wenn die zwei Sätze parataktisch verbunden wären. Dass die Struktur der beiden Nebensätze in (30) das Ergebnis einer persönlichen Einstellung des Sprechers ist, ersieht man auch aus der Tatsache, dass der Wahrheitswert keiner der zwei Sätze von einer folgenden Aussage geschmälert werden darf, wie (31) illustriert:

(31) a. ?Bei uns ist ein Mann, der redet drauflos. Aber ich bin mir nicht sicher, ob das stimmt.

b. ?Das ist Musik, die wird nicht sterben. Zumindest sagt man das so.

Die VL-Varianten der Sätze in (31) sind pragmatisch akzeptabler, was zeigt, dass der Sachverhalt der Relativsätze mit V2-Wortstellung nicht nur neuer und informativer ist als der des Matrixsatzes, sondern auch assertiert wird und eine persönlichen Meinung bzw. Einstellung des Sprechers ausdrückt. Der Nebensatz enthält also in der Regel eine Information, die der Sprecher für überraschend bzw. relevant in dem Kontext hält und auf die die Aufmerksamkeit des Gesprächspartners gelenkt werden soll. Diese sprecher-orientierte Information wird assertiert (dies rechtfertigt im Übrigen auch strukturell die V2Wortstellung), was andeutet, dass der Sprecher, unabhängig davon, ob dieser Sachverhalt eine Entsprechung in der empirischen Realität hat, aus seiner Perspektive überzeugt vom übermittelten Inhalt ist. Die konkreten Implikationen der Darstellung des Sprechers in solchen Konstruktionen hängen natürlich vom spezifischen Kontext ab: Es handelt sich bei V2Relativsätzen aber in allen Fällen um einen Inhalt, den der Sprecher wesentlich in dem Kontext findet, wo er erscheint. Manche Formen der V2-Relativsätze haben sich in der Sprache sogar herauskristallisiert: Z. B. kommen V2-Relativsätze oft in den einleitenden Formeln von Märchen vor, wie (32) zeigt:

a. Es war einmal ein Mann, der hatte einen hohlen Zahn. In dem Zahn war ein Kistchen drin. In dem Kistchen war ein Zettel. Auf dem Zettel stand "es war einmal ein Mann, der hatte einen hohlen Zahn $[\ldots]^{\prime 2} .{ }^{22}$

b. Es war einmal ein König, der hatte drei Söhne. Als er sein Ende nahen fühlte, geriet er in Sorge, welchem seiner Söhne er wohl sein Reich vererben könnte [...]. ${ }^{23}$

In diesen beiden Fällen (das zweite Beispiel wurde übrigens aus der semi-spontanen sprachlichen Produktion eines 11 Jahre alten Kindes entnommen) zeigt sich deutlich die Distribution der Informationen im Satz, die oben anhand anderer Beispiele erwähnt wurde: Der Nebensatz hat eine V2-Wortstellung, weil die in der eingebetteten Struktur geäußerte Information (jeweils: Dass der Mann einen hohlen Zahn hatte [32a] und dass der König drei

22 Aus: http://zenoparadox.wordpress.com/2011/03/12/warum-metadata-strategien-scheitern/, Warum MetadataStrategien scheitern, 11.03.2011, Stand: 19.12.2011. 
Söhne hatte [32b]) laut dem Sprecher die Basis für die Fortführung des Märchens bildet. Dass solche Strukturen spezifisch in dieser Texttypologie erscheinen und sich auch im geschriebenen Gebrauch herauskristallisiert haben, ist tatsächlich kein Zufall, denn Fabeln und Märchen sind Texte, die typischerweise mündlich übertragen werden. In dem situationellen Kontext der Erzählung eines Märchens kann der Erzähler natürlich die Informationen syntaktisch sowie prosodisch betonen, die er als die relevantesten präsentieren will.

\section{Schlussbemerkungen}

Die bisher vorgelegten Annahmen haben selbstverständlich keine Ambition, endgültige Antworten auf die oben angedeuteten Fragen zu liefern. Der theoretische Ausgangspunkt, der hier nur skizziert wurde, soll nur als Orientierung gelten (besonders für die Annahme, dass die drei untersuchten V2-Satztypen eine Hypotaxe und nicht eine Parataxe mit dem unmittelbar benachbarten Satz bilden und dass das V2-Phänomen bedeutsame Auswirkungen auf die Semantik-Pragmatik der Äußerung hat) und muss durch die Vertiefung bestimmter Aspekte der V2-Einbettung - auch von einer vergleichenden Perspektive aus - integriert werden. Das Phänomen der V2-Einbettung bei Relativsätzen ist gewissermaßen kontroverser als bei weilund dass-Sätzen, denn im Deutschen sind Relativpronomina Homophone mit Demonstrativa (und tragen wie Demonstrativpronomina die Genus- und Numerus-Merkmale der im Hauptsatz vorkommenden DP, während ihr Kasus vom Verb des Satzes, wo sie erscheinen, bestimmt wird), was intuitiv zur Schlussfolgerung führen könnte, dass es sich bei solchen Strukturen um Hauptsätze handelt, die parataktisch verbunden mit dem "ehemaligen" Hauptsatz (d. h. mit dem Satz, der dem d-Satz unmittelbar vorausgeht) sind. Es wurde aber in dieser Arbeit gezeigt (wie Gärtner [2002: 35] vorschlägt), dass solche Strukturen als "echte" Relativsätze konzipiert werden sollen. Was gezeigt wurde, ist, dass die von Gärtner (2001) und anderen Autoren vorgeschlagene syntaktische Analyse der inneren Struktur "nichtkanonischer" V2-Relativsätze nicht überzeugend ist, und zwar auf der Basis von Argumenten unterschiedlicher Natur: (i) die prosodische Kontur von Strukturen des Deutschen, die von einem d-Element eingeleitet werden, weist in V2- und VL-Sätzen keine besondere Variation auf, d.h. aus phonologischer Perspektive unterscheiden sich die zwei Typen nicht; (ii) besondere Instanzen dieser Konstruktion (vgl. z. B. (4), (5), (8), (9)) zeigen eindeutig, dass die logisch-semantische Interpretation eines V2-Relativatzes nicht parataktisch sein kann, denn eine Koordination impliziert zwei Assertionen und das ist bei vielen der hier angeführten Beispiele nicht der Fall; (iii) die Produktion der V2-Relativsätze scheint dadurch motiviert zu sein, dass der Sprecher mittels des V2-Nebensatzes einen Inhalt zum Ausdruck bringt, der "assertiert" wird, oder genauer gesagt eine emotionale Verwicklung des Sprechers involviert und informationsstrukturell neuer und relevanter ist als der des Hauptsatzes.

\section{Literatur}

Antomo, Mailin/Markus Steinbach (2010): "Desintegration und Interpretation. Weil-V2-Sätze an der Schnittstelle zwischen Syntax, Semantik und Pragmatik". Zeitschrift für Sprachwissenschaft 29: 1-37.

Antomo, Mailin (2012): "Interpreting Embedded Verb Second. Causal Modifiers in German". In: Costantinescu, Camelia et al. (ed.) (2012): Proceedings of ConSOLE XVII: 27-51. 
Ballweg, Joachim et al. (1997): Grammatik der deutschen Sprache. Berlin/New York: Mouton de Gruyter.

Birkner, Karin (2008): Relativ(satz)konstruktionen im gesprochenen Deutsch. Syntaktische, prosodische, semantische und pragmatische Aspekte. Berlin/New York: de Gruyter.

Blühdorn, Hardarik (2007): "Zur Struktur und Interpretation von Relativsätzen". Deutsche Sprache 35/4: 287-314.

Brandt, Margareta (1990): Weiterführende Nebensätze: Zu ihrer Syntax, Semantik und Pragmatik. Stockholm: Almqvist \& Wiksell. (=Lunder germanistische Forschungen 57).

Curme, George O. (1922): A Grammar of the German Language. New York: Frederick Ungar.

Den Besten, Hans (1977/1983): "On the Interaction of Root Transformations and Lexical Deletive Rules". In: Abraham, Werner (ed.) (1977/1983): On the Formal Syntax of the Westgermania. Amsterdam, John Benjamins: 47-131.

Den Dikken, M. (2005): "A Comment on the Topic of Topic-Comment". Lingua 115: 691710.

Ebert, Christian/Endriss, Cornelia/Gärtner, Hans-Martin (2007): "An Information Structural Account of German Integrated Verb Second Clauses". Research on Language and Computation 5/4: 415-434.

Endriss, Cornelia/Hans-Martin Gärtner (2005): "Relativische Verbzweitsätze und Definitheit". In: D'Avis, Franz-Josef (ed.) (2005): Deutsche Syntax: Empirie und Theorie. Göteborg: Acta Universitatis Gothoburgensis: 195-220.

Franco, Irene (2010): "Issues in the Syntax of Scandinavian Embedded Clauses". Lund University Working Papers in Scandinavian Syntax 86: 137-177.

Freywald, Ulrike (2008): "Zur Syntax und Funktion von dass-Sätzen mit Verbzweitstellung". Deutsche Sprache 36: 246-285.

Freywald, Ulrike (2009): "Kontexte für nicht-kanonische Verbzweitstellung: V2 nach dass und Verwandtes". In: Ehrich, Veronika/Fortmann, Christian/Reich, Ingo/Reis, Marga (eds.) (2009): Koordination und Subordination im Deutschen. Hamburg, Buske: 113-134.

Fritsch, Werner Johann (1990): Gestalt und Bedeutung der deutschen Relativsätze. Dissertation, Ludwig-Maximilians-Universität München.

Frosch, Helmut (1996): "Appositive und restriktive Relativsätze". Sprachtheorie und germanistische Linguistik 2: 7-19.

Gärtner, Hans-Martin (2001): "Are there V2 Relative Clauses in German?" The Journal of Comparative Germanic Linguistics 3: 97-141.

Gärtner, Hans-Martin (2002): "On the Force of V2 Declaratives". Theoretical Linguistics 28: $33-42$.

Gärtner, Hans-Martin/Michaelis, Jens (2010): "On Modeling the Distribution of Declarative V2-Clauses: the Case of Disjunction". In: Bab, Sebastian/Robering, Klaus (eds.) (2010): Judgements and Propositions. Berlin, Logos Verlag: 11-25.

Gaumann, Ulrike (1983): "Weil die machen jetzt bald zu". Angabe- und Junktivsatz in der deutschen Gegenwartssprache. Göppingen: Kümmerle.

Günthner, Susanne (1996): "From Subordination to Coordination? Verb-second Position in German Causal and Concessive Constructions". Pragmatics 6/3: 323-356. 
Iatridou, Sabine/Anthony Kroch (1992): "The Licensing of CP-Recursion and its Relevance to the Germanic Verb-Second Phenomenon". Lund University Working Papers in Scandinavian Syntax 50: 1-24.

Julien, Marit (2007): "Embedded V2 in Norwegian and Swedish". Lund University Working Papers in Scandinavian Syntax 80: 103-161.

Krifka, Manfred (2006): Dimensionen grammatischer Variation: Eine Einführung in die Sprachtypologie. Handout Humboldt Universität, Berlin, Wintersemester 2006/2007.

Lee, Mi-Young (2012): "Das Rätsel von SVO beim Erlernen des Deutschen - Warum ist SVO so leicht, SOV dagegen so schwer produzierbar?". Zeitschrift für interkulturellen Fremdsprachenunterricht 17/1: 75-92.

Ravetto, Miriam (2006): Le "false relative" in tedesco: Es war einmal ein Königssohn, der bekam Lust in der Welt umher zu ziehen. Vercelli: Mercurio.

Reis, Marga (1997): "Zum syntaktischen Status unselbständiger Verbzweit-Sätze". In: D'Avis, Franz-Josef/Lutz, Uli (eds.) (1997): Zur Satzstruktur des Deutschen - Arbeitspapiere des SFB 340/90. Stuttgart/Tübingen: 121-142.

Sandig, Barbara (1973): "Zur historischen Kontinuität diskriminierter syntaktischer Muster in spontaner Sprechsprache". Deutsche Sprache 1: 37-57.

Sturm, Afra (2005): Eigennamen und Definitheit. Tübingen: Niemeyer.

Stechov, Arnim, Von (2007): Schritte zur Satzsemantik. http://www.sfs.unituebingen.de/ astechow/Lehre/WS06_7/SemantikII/ASkript/14_RelativPRO.pdf, Stand: 4. August 2014.

Sternefeld, Wolfgang (1990): Syntaktische Grenzen. Eine kritische Darstellung der Barrierentheorie Noam Chomskys. Groningen: Rijksuniv. (= GAGL 31).

Sternefeld, Wolfgang (2004): Eine merkmalbasierte generative Analyse des Deutschen. Tübingen: Stauffenburg. (= Stauffenburg Linguistik 31).

Willems, Klaas (1994): "Weil es hat mit Bedeutung nicht viel zu tun. Zum Sprachwandel einer Konjunktion". Deutsche Sprache 22: 261-279. 